\title{
Transpolar potential saturation models compared
}

\author{
G. Siscoe \\ Center for Space Physics, Boston University, Boston, Massachusetts, USA
}

J. Raeder

Space Science Center and Department of Physics, University of New Hampshire, Durham, New Hampshire, USA

\section{A. J. Ridley}

Space Physics Research Laboratory, University of Michigan, Ann Arbor, Michigan, USA

Received 11 November 2003; revised 24 March 2004; accepted 18 June 2004; published 8 September 2004.

[1] We compare four explanations of transpolar potential saturation: (1) the magnetic field at the stagnation point weakens, thereby limiting magnetic reconnection; (2) a dimple develops at the stagnation point, which limits the inflow rate to the reconnection line; (3) the magnetopause becomes blunt and the bow shock recedes, thus giving more room for the solar wind to flow around the magnetosphere, thereby reducing the need for magnetic reconnection; (4) the region 1 current system usurps the Chapman-Ferraro current system and saturates when the $\mathbf{J} \times \mathbf{B}$ force it generates balances solar wind ram pressure. The paper's point is that all four mechanisms involve a limit on the strength of the region 1 current system and that the criterion for the onset of transpolar potential saturation in each mechanism is that the region 1 current system generates a magnetic field that is about as strong as the dipole field at the dayside magnetopause. This circumstance prevents tests to discriminate between the four mechanisms based on predictions that relate to their dependencies on the region 1 current system. The group as a whole, however, can be tested to see whether their common criterion that relates the onset of transpolar potential saturation to the total current flowing in the region 1 system holds. The criterion can be formulated in terms of predictions that relate transpolar potential saturation to the strength of the interplanetary electric field, solar wind ram pressure, and ionospheric conductance. Published data analyses and MHD simulations reasonably confirm these predictions. INDEX TERMS: 2740 Magnetospheric Physics: Magnetospheric configuration and dynamics; 2708 Magnetospheric Physics: Current systems (2409); 2736 Magnetospheric Physics: Magnetosphere/ionosphere interactions; KEYWORDS: polar cap potential, saturation, polar cap potential saturation, cross-polar potential saturation, transpolar potential saturation

Citation: Siscoe, G., J. Raeder, and A. J. Ridley (2004), Transpolar potential saturation models compared, J. Geophys. Res., 109, A09203, doi:10.1029/2003JA010318.

\section{Transpolar Potential Saturation}

[2] We call "transpolar potential" the difference between the maximum and minimum voltages associated with ionospheric convection that the solar wind drives at high latitudes through its coupling to the magnetosphere. Saturation of the transpolar potential refers to its tendency to level off instead of rising linearly as solar wind driving increases from weak to strong (quantified below). Most of the time the strength of solar wind driving stays within the weak range for which the response of the transpolar potential is linear. Transition into the saturated range occurs at times associated with magnetic storms, which are somewhat rare events. Consequently, we have relatively few measurements taken while the transpolar po-

Copyright 2004 by the American Geophysical Union. 0148-0227/04/2003JA010318 tential is saturated. Nonetheless, data of various types from which the transpolar potential in the saturation range can be obtained have accumulated so that now evidence of the phenomenon is sufficiently convincing. Recent examples include assimilated mapping of ionospheric electrodynamics (AMIE) reconstructions of ionospheric potentials [Russell et al., 2000, 2001; Liemohn et al., 2002], DMSP drift meter measurements [Hairston et al., 2003], high-latitude radar reconstructions of synoptic-scale ionospheric flows [Shepherd et al., 2002], and a calibration of a polar cap magnetic index in terms of ionospheric convection electric field strength [Tsutomu, 2002].

[3] Further evidence comes from global MHD simulations of the magnetosphere, which can probe the saturation range of solar wind driving strengths at will. A number of MHD simulation studies showing the saturation phenomenon have been reported [Raeder et al., 2001; Siscoe et al., 2002a; Raeder and Lu, 2004; Merkine et al., 2003] and to 


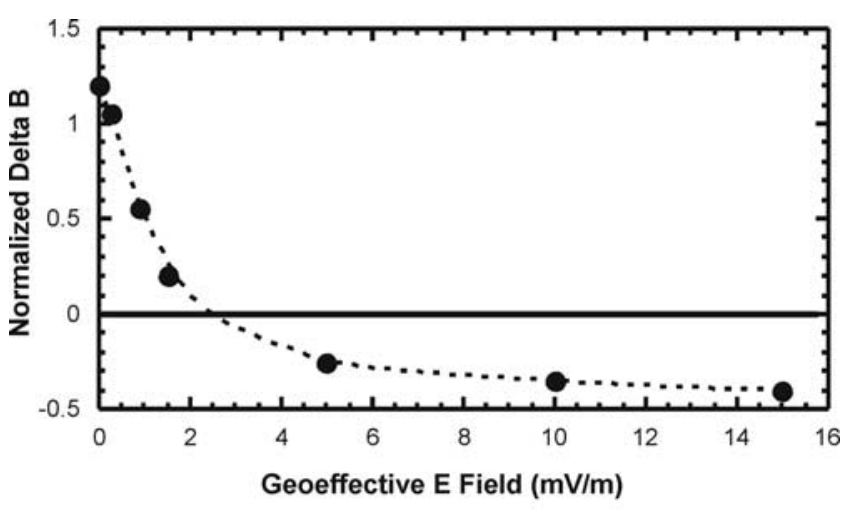

Figure 1. Deviation from the dipole value of the magnetic field strength at the stagnation point as a function of interplanetary electric field (southward interplanetary magnetic field (IMF)) as computed by MHD simulations. Values normalized to dipole strengths and shifted so that zero corresponds to the dipole value (from Siscoe et al. [2002b]).

the authors' knowledge every MHD code that has looked for the saturation phenomenon has found it.

\section{Explanations of Transpolar Potential Saturation}

[4] We compare four seemingly different ideas that have been proposed to account for the phenomenon of transpolar potential saturation: (1) dipole field reduction at the subsolar magnetopause [Hill et al., 1976]; (2) dimple formation at the stagnation point [Ridley, 2001; Raeder et al., 2001]; (3) widening of the magnetosheath which relieves the tendency to force reconnection at the magnetopause (V. G. Merkine et al., Relationship between ionospheric conductance, field aligned current, and magnetopause geometry: Global MHD simulations, submitted to Geophysical Research Letters, 2004, hereinafter referred to as Merkine et al., submitted manuscript, 2004); (4) ram pressure saturation [Siscoe et al., 2002b]. All four mechanisms entail a role for the region 1 current system, the geometry of which (based on global MHD simulations) is envisioned to be two loops (one in each hemisphere) that close at high altitude over the poles [Tanaka, 1995; Janhunen et al., 1996; Siscoe et al., 2000]. Transpolar potential saturation entails increasing the total current in the region 1 current system until something happens that limits further strengthening. By limiting the total region 1 current, one also limits the transpolar potential though the ionospheric Ohm's law, thus accounting for transpolar potential saturation. The four mechanisms listed above differ in their descriptions of the process that halts the strengthening of the total current in the region 1 current system beyond some limit. The role of the region 1 current system can be viewed alternatively as a cause of the saturation mechanism or as a necessary concomitant of the mechanism. The distinction is unimportant for the present discussion.

[5] The list does not include the mechanism proposed by Winglee et al. [2002] in which mass loading from ionospheric outflow is invoked to offer inertial resistance to transpolar convection and hence to reduce the rate of increase of the transpolar potential as the interplanetary electric field (IEF) increases. Perhaps this mechanism also can be related to the current that flows in the region 1 current system, but the relation is not as direct as it is for the four mechanisms listed above. Therefore since we cannot treat it as one of the others, we shall omit it from the present discussion. It reenters in section 5 after tests have been applied to the listed four mechanisms.

[6] The first of the listed mechanisms (the Hill mechanism) invokes a limit on the strength of the region 1 system resulting from its property to generate a magnetic field that opposes the dipole field at the magnetopause at low latitudes. There is therefore an obvious upper limit on the strength of the region 1 system, namely, that which would cancel the dipole field on the magnetospheric side of the stagnation point. Since this limit is impossible to reach, because it would, in effect, remove the obstacle, the limit is put instead at some fraction (given below) of the dipole field strength at the stagnation point.

[7] To find support for the assertion that the field is significantly weakened at the stagnation point under conditions associated with transpolar potential saturation, we look to MHD simulations. Figure 1 shows field strength at the stagnation point as computed by MHD simulations as a function of the driving IEF. Field strength is presented as $\mathrm{B} / \mathrm{B}_{\mathrm{D}}-1$, which is the field strength normalized to the dipole field strength and shifted such that the zero line denotes no deviation from dipole field strength. The figure shows that field strength decreases as the IEF increases from unsaturated to saturated levels, as the Hill mechanism assumes. The curve indicates that the strength of the field at the stagnation point is actually less than the dipole field when the IEF associated with a southward interplanetary magnetic field (IMF) exceeds $\sim 3 \mathrm{mV} / \mathrm{m}$. For example, at geosynchronous orbit the dipole field strength is close to $108 \mathrm{nT}$. If pure compression were to push the stagnation point in to this distance, the compressed field strength would be about $238 \mathrm{nT}$, a little more than twice the dipole field. If, however, the IEF of a southward IMF were $5 \mathrm{mV} / \mathrm{m}$, then, as one can infer from the numbers given in the figure, the field strength at the stagnation point instead of being $\sim 238 \mathrm{nT}$ would be $\sim 81 \mathrm{nT}$, that is, $\sim 25 \%$ less than the dipole field. Such weakening has been reported. Cahill and Winckler [1999] gives a plot of magnetic field strength measured by GOES 6 in the noon meridian as the magnetopause passed inward over it during the storm of 8 November 1991. The field strength measured at the magnetopause instead of being more than twice the dipole field strength was $\sim 10 \mathrm{nT}$ less than it. Thus the weak field assumption of mechanism 1 appears to hold.

[8] The thought behind the second of the listed mechanisms (dimple formation at the stagnation point) is that as the region 1 system strengthens, it generates a perturbation magnetic field that produces a dimple in the magnetopause at or near the low-latitude site of magnetic reconnection (shown below). This happens for the same reason just mentioned in connection with the Hill mechanism. The region 1 system weakens the magnetic field at the dayside magnetopause at low latitudes (the idea behind the Hill mechanism), but at the same time it intensifies the field at high latitudes at the dayside magnetopause. Thus the magnetopause develops high-latitude shoulders and a low- 


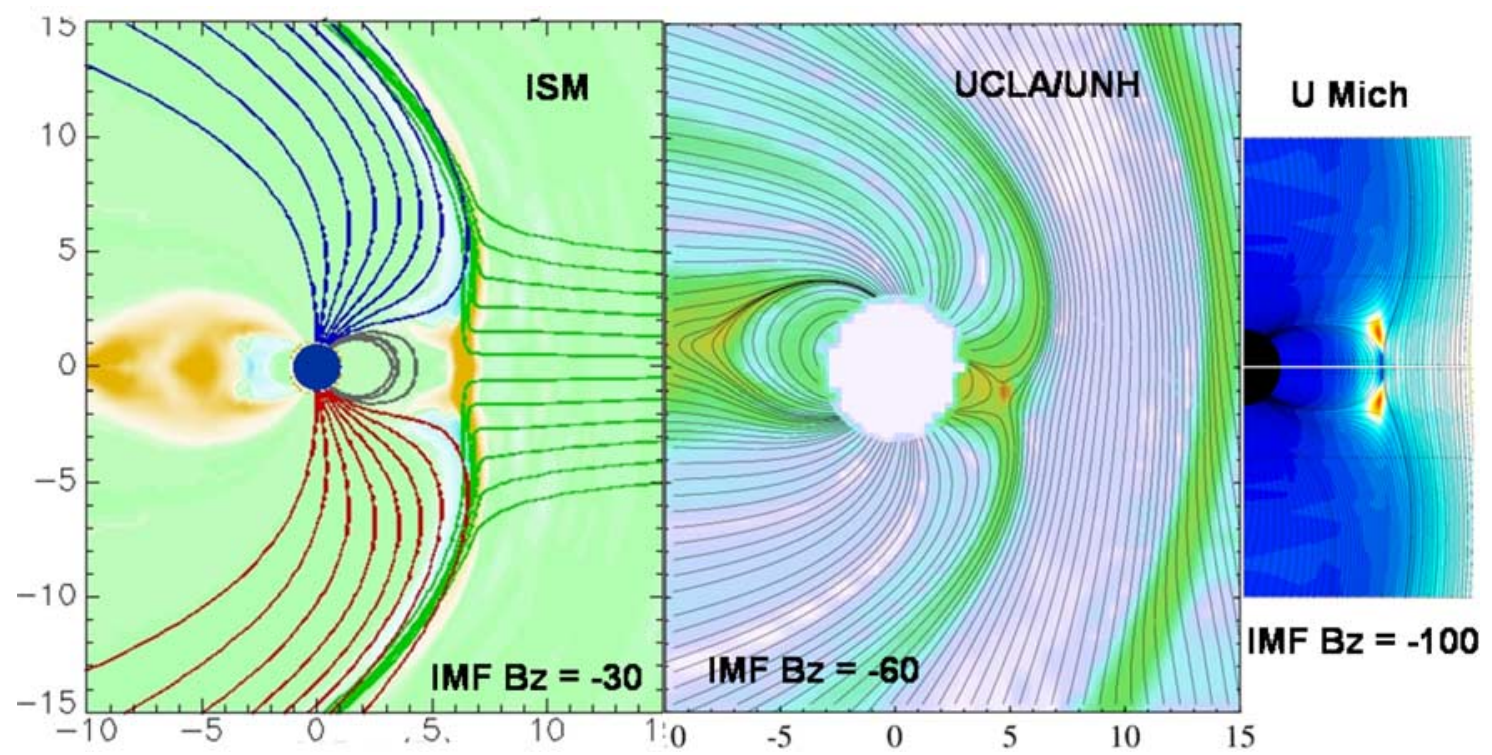

Figure 2. Three MHD simulations showing the dimple that develops in the magnetopause in the noonmidnight meridian plane under saturated conditions. The panels represent results from three different MHD codes and for increasing degrees of saturation (left to right). Format varies between panels: Left shows electric current contours and solar wind streamlines; middle shows pressure contours and magnetic field lines; and right shows velocity contours and magnetic field lines.

latitude dimple. Dimples tend to fill with stagnated plasma, which puts a buffer between the boundary and the flow [e.g., Spreiter and Summers, 1967]. Consequently, as a dimple begins to form, the solar wind might begin to lose its reconnection grip on the magnetopause, thus halting further increase of convection.

[9] An alternative view of dimple formation comes from vacuum field superposition. If one superposes a uniform southward field on the Earth's dipole field, the geometry of field lines around the equatorial null line, where in the real case reconnection occurs, can be described as dimple-like. The point is that dimple geometry is natural to the system when the IMF is directed southward. Dimple geometry does not typically happen in the real case, however, because the Chapman-Ferraro field compresses the dipole field and, in effect, presses out the dimple. However, under saturation conditions, Chapman-Ferraro compression goes away (shown below), so the vacuum dimple geometry can express itself to some extent.

[10] MHD simulations support the dimple mechanism. Figure 2 shows the characteristic shape that develops in the magnetopause under saturation conditions as computed by three different MHD codes. The left panel shows results from the ISM MHD code [White et al., 2001] for a straight southward, $30 \mathrm{nT}$ IMF. The middle panel shows results from a simulation using the University of California, Los Angeles (UCLA)/University of New Hampshire (UNH) MHD code of the Bastille Day storm [Raeder et al., 2001]. In the simulation the dipole was tilted to represent the solstice situation. During this storm the IMF reached $-60 \mathrm{nT}$ while the solar wind velocity was $1100 \mathrm{~km} / \mathrm{s}$. The third panel shows an extreme case simulated with the University of Michigan code [Ridley, 2003]. The interplanetary electric fields are $12 \mathrm{mV} / \mathrm{m}, 66 \mathrm{mV} / \mathrm{m}$, and $40 \mathrm{mV} / \mathrm{m}$, respectively. The high-latitude shoulders and low-latitude dimple of the magnetopause are evident. The three images indicate qualitatively how the shape of the magnetopause might be self-limiting, since the flow must reach the stagnation point and be ejected to allow the shape to evolve. Referring to the left panel, the solar wind streamlines shown there indicate how the shoulders and dimple cause the flow to become congested as it leaves the stagnation point. The right panel makes the same point by showing that the reconnection jets (the red features) are very short compared with those that form for smaller values of southward IMF $\mathrm{Bz}$ (not shown). In every case, a dimple clearly develops at the reconnection point, which means that as far as MHD simulations are concerned, the basic prerequisite for mechanism 2 is satisfied.

[11] The third mechanism in the list is a variation on the magnetopause distortion theme in which the magnetic field that the region 1 current system generates makes the shape of the magnetosphere more blunt. In consequence the standoff distance of the shock increases, an effect that is augmented by the Mach number of the solar wind usually decreasing at times associated with transpolar potential saturation. An increased standoff distance allows more room for the postshock flow to brake and flow around the magnetosphere, which reduces the need for magnetopause reconnection to aid the process [Merkine et al., 2003; Merkine et al., submitted manuscript, 2004]. (Although we are emphasizing the role of the region 1 current system in the blunt magnetopause mechanism, the emphasis of the Merkine et al. works is on an increase in ionospheric conductance as the proximate cause of transpolar potential saturation. These are mutually consistent viewpoints, however. Our emphasis on region 1 currents instead of ionospheric conductance merely illustrates the interdependence of the coupled magnetosphere-ionosphere system. If an increase in ionospheric conductance is the true cause of 


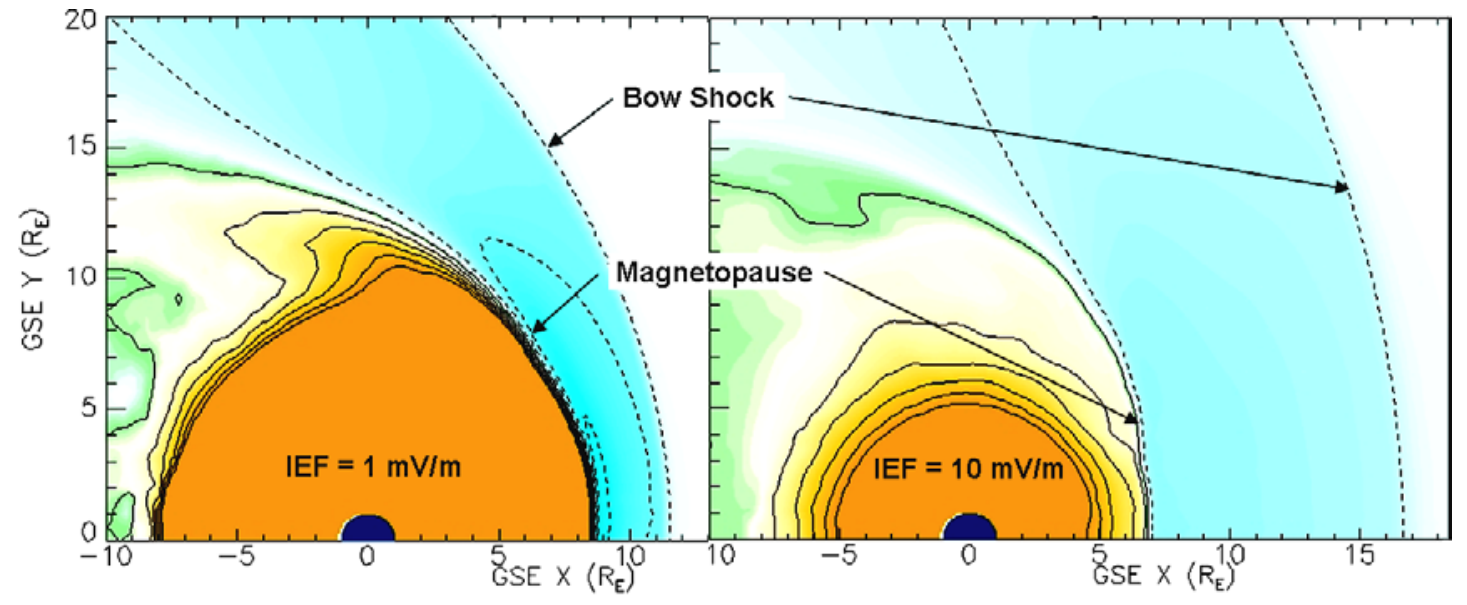

Figure 3. Contours in the equatorial plane of the north-south component of the magnetic field show the shapes of the magnetopause (green contour) and the bow shock (as labeled) under conditions associated with unsaturated (left) and saturated (right) transpolar potentials. Tan areas mark positive values, and blue areas mark negative values. (Images were generated using output from the ISM code and the Weimer graphics program ISM-VIEW.)

transpolar potential saturation, it is nonetheless the resulting increase in region 1 current that correlates with the change in shape of the magnetopause and the correlated lessening of reconnection.)

[12] Again we look to MHD simulations to find evidence that the prerequisite shape change of mechanism 3 occurs. Figure 3 depicts the magnetopause and bow shock in the equatorial plane. The two panels show unsaturated (IEF = $1 \mathrm{mV} / \mathrm{m}$ ) and saturated (IEF $=10 \mathrm{mV} / \mathrm{m}$ ) situations for a solar wind speed and density of $500 \mathrm{~km} / \mathrm{s}$ and 5 protons $/ \mathrm{cm}^{3}$. The strength of the IMF is $-2 \mathrm{nT}$ on the left and $-20 \mathrm{nT}$ on the right. The contours in the figure are lines of constant strength of the north-south component of the magnetic field. Contours are separated by $4 \mathrm{nT}$ on the left and $40 \mathrm{nT}$ on the right. The zero level is marked by a green contour, which separates northward pointing field (colored tan) inside the magnetosphere from southward pointing field (colored blue) in the magnetosheath. Thus the green contour marks the magnetopause.

[13] That the magnetopause on the right is blunter than the one on the left can be seen by noting that the x-position of the nose is $1.5 \mathrm{Re}$ closer to Earth on the right than on the left while the y-positions of the magnetopause in the terminator plane $(\mathrm{x}=0)$ are virtually the same $(12.5 \mathrm{Re})$ in both cases. For this reason, in part, the bow shock moves out. However, another part of the reason that the bow shock moves out is that in the simulation the Alfvén Mach number of the solar wind is about three times smaller on the right than on the left. Both effects, blunter magnetopause and smaller Mach number, probably also operate in nature to increase the bow shock standoff distance under conditions associated with transpolar potential saturation. Thus the scenario of mechanism 3 finds support in MHD simulations.

[14] The fourth of the listed mechanisms (ram pressure limitation) draws on the property of the region 1 current system to usurp and ultimately to replace the ChapmanFerraro current system. Since there is a hard upper limit on the Chapman-Ferraro current set by the requirement to balance the total solar wind ram pressure, the same limit is assumed to apply to the region 1 system when it takes over. As a prerequisite for this to happen, there should be no current that closes entirely on the magnetopause, which is the distinguishing geometrical characteristic of the Chapman-Ferraro current system. Instead, the magnetopause should consist entirely of current that closes in the ionosphere as region 1 currents. Figure 4 illustrates that this prerequisite is met in MHD simulations.

[15] For elaboration on these mechanisms, the reader is asked to consult the cited references. The object here is to

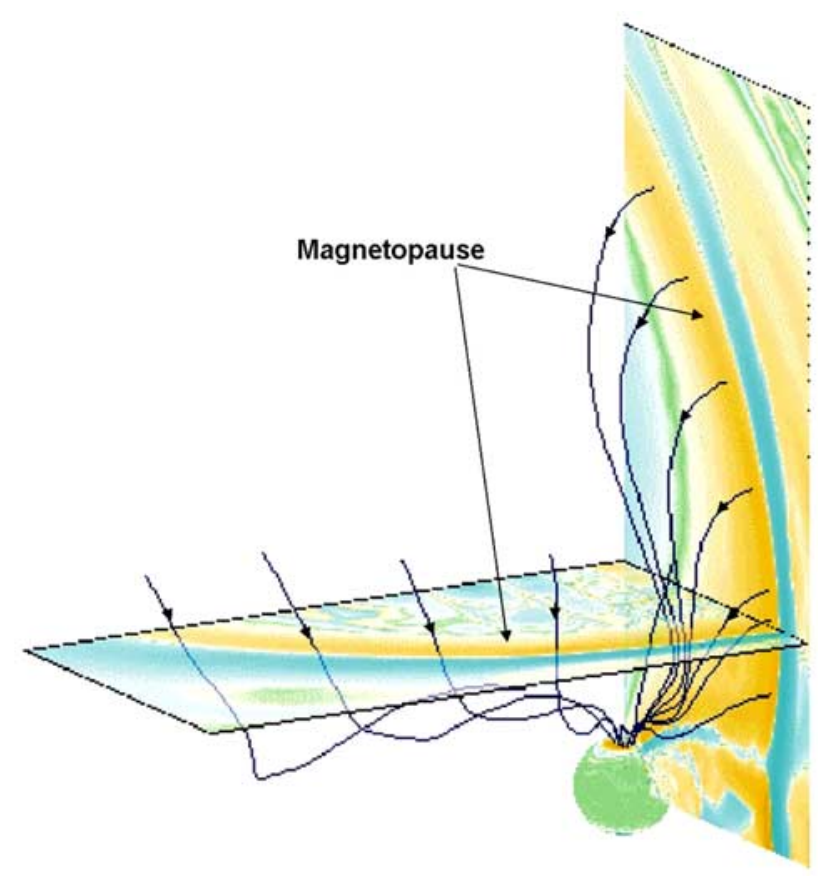

Figure 4. Contours of magnetic force $(\mathbf{J} \times \mathbf{B})$ and current streamlines. Tan areas mark outward force (hence the magnetopause). Note that all current streamlines go to the ionosphere as region 1 currents. (Images were generated using output from the ISM code and the Weimer graphics program ISM-VIEW.) 
point out that because of their connection to the region 1 current system (direct or indirect does not matter), the criterion for the onset of saturation is essentially the same for each mechanism, as the next section attempts to make clear.

\section{Commonality of Saturation Criterion}

[16] To repeat the central point, in all mechanism under review the transpolar potential entering the saturation domain corresponds to the total current in the region 1 system rising to a level at which the mechanism to which it is connected changes from being unimportant to being dominant. The level to which the region 1 current rises when the transpolar potential enters the saturation domain can be seen to be about the same for each mechanism. Consider first the Hill mechanism, which says that saturation happens when the region 1 current system generates a magnetic field at the stagnation point that significantly weakens the dipole field there. What "significant weakening" means in this case can be bracketed. Since saturation is not a small effect (it dominates the electrodynamic coupling between the solar wind and the ionosphere), the weakening cannot be small, say a few percent. Also obviously it cannot be $100 \%$, which would effectively eliminate the Earth as seen by the solar wind. Given only this much information, an unbiased value should be $\sim 50 \%$, which is neither inappropriately small nor impossibly large and which favors neither extreme. Therefore in the absence of knowledge of the precise degree of reduction needed for the mechanism to operate, we associate saturation in the Hill mechanism with the region 1 current system generating a magnetic field at the stagnation point that is about $1 / 2$ the strength of the dipole field there.

[17] Consider next the "dimple formation" mechanism. Presumably, like the Hill mechanism just discussed, the formation of a dimple at the magnetopause may be expressed in terms of the region 1 current system generating a magnetic field at the stagnation point that is a certain fraction between 0 and 1 of the dipole field there. Recall that a dimple forms because the field that the region 1 current system generates weakens the field at low latitudes on the dayside but strengthens it at high latitudes, thus producing high-latitude shoulders and an equatorial dimple. How much region 1 current is required to bring about this effect? Again for the same reason as in the Hill mechanism, since we do not know the actual fraction of the dipole strength to use and since we are not dealing with subtle effects, the a priori, unbiased value to choose is once more $1 / 2$.

[18] The pattern of argument established in discussing the Hill and dimple-formation mechanisms applies to the magnetopause-blunting mechanism as well. Recall that here the region 1 current system must generate a field at the magnetopause strong enough to significantly alter its shape (Merkine et al., submitted manuscript, 2004). The pertinent point is that to alter the shape of the magnetopause the region 1 field must be comparable in strength to the field that was there without it. So again for saturation the region 1 field must be a significant fraction of the dipole field at the magnetopause, and thus here too a reasonable a priori, unbiased criterion is that the region 1 field be $1 / 2$ the dipole at the stagnation point.
[19] Last in the list is the ram pressure mechanism. It assumes that saturation happens when the region 1 current system replaces the Chapman-Ferraro current system completely and thereby takes sole responsibility for deflecting the solar wind around the magnetosphere. At this point the total current in the region 1 system has become as large as the interaction with the solar wind allows. To estimate the strength of the magnetic field that the region 1 system generates at the stagnation point when this happens, we should ask what fraction of the dipole field the ChapmanFerraro system ordinarily generates there. The answer is $\sim 1$. So this should be about what the region 1 system produces when it takes over, except that instead of approximately doubling the dipole field, as does the Chapman-Ferraro system, the region 1 system would approximately cancel it. Thus the situation in this regard closely resembles the Hill mechanism and as in that case the unbiased choice of fractional weakening between 0 and 1 that the region 1 provides is $1 / 2$ the dipole field at the stagnation point.

[20] We conclude that all four mechanisms, though apparently different in terms of the physics to which they appeal, are nonetheless about the same when represented quantitatively in terms of an explicit requirement on the total current in the region 1 system associated with saturation. The quantitative value $1 / 2$ with which we have identified the condition of saturation might change when the descriptions of the mechanisms mature. Then it might be possible to discriminate between the mechanisms by means of this criterion. Meanwhile, we can use the common criterion for saturation to devise tests that apply to the four mechanisms as a group. For this purpose we use the Hill mechanism to represent the group because it has been modeled analytically to give the dependence of the value of the saturated potential $\left(\Phi_{\mathrm{S}}\right)$ on interplanetary electric field (IEF), solar wind ram pressure $(\mathrm{P})$, and ionospheric conductance $(\Sigma)$, as the next section describes.

\section{Hill Equation for Transpolar Potential Saturation}

[21] Hill postulated a mathematical expression that embodies the essential characteristics of transpolar potential saturation [Hill et al., 1976]. In basic form, Hill's expression is

$$
\Phi_{T}=\frac{\Phi_{L} \Phi_{S}}{\Phi_{L}+\Phi_{S}}=\frac{\Phi_{L}}{1+\frac{\Phi_{L}}{\Phi_{S}}},
$$

where $\Phi_{\mathrm{T}}$ is the transpolar potential, $\Phi_{\mathrm{L}}$ is the form of the potential that varies linearly with the strength of solar wind driving, and $\Phi_{\mathrm{S}}$ is the saturated value of the transpolar potential. Note that when $\Phi_{\mathrm{L}} \ll \Phi_{\mathrm{S}}$ (weak driving), $\Phi_{\mathrm{T}}=\Phi_{\mathrm{L}}$ (the linear domain), and when $\Phi_{\mathrm{L}} \gg \Phi_{\mathrm{S}}$ (strong driving), $\Phi_{\mathrm{T}}=\Phi_{\mathrm{S}}$ (the saturated domain). This formulation therefore captures the central behavior of transpolar potential saturation.

[22] Siscoe et al. [2002b] gives expressions for $\Phi_{\mathrm{L}}$ and $\Phi_{\mathrm{S}}$ in terms of IEF, P, and $\Sigma$ :

$$
\Phi_{\mathrm{L}}(\mathrm{kV})=57.6 \operatorname{IEF}(\mathrm{mV} / \mathrm{m}) \mathrm{P}(\mathrm{nPa})^{-1 / 6}
$$




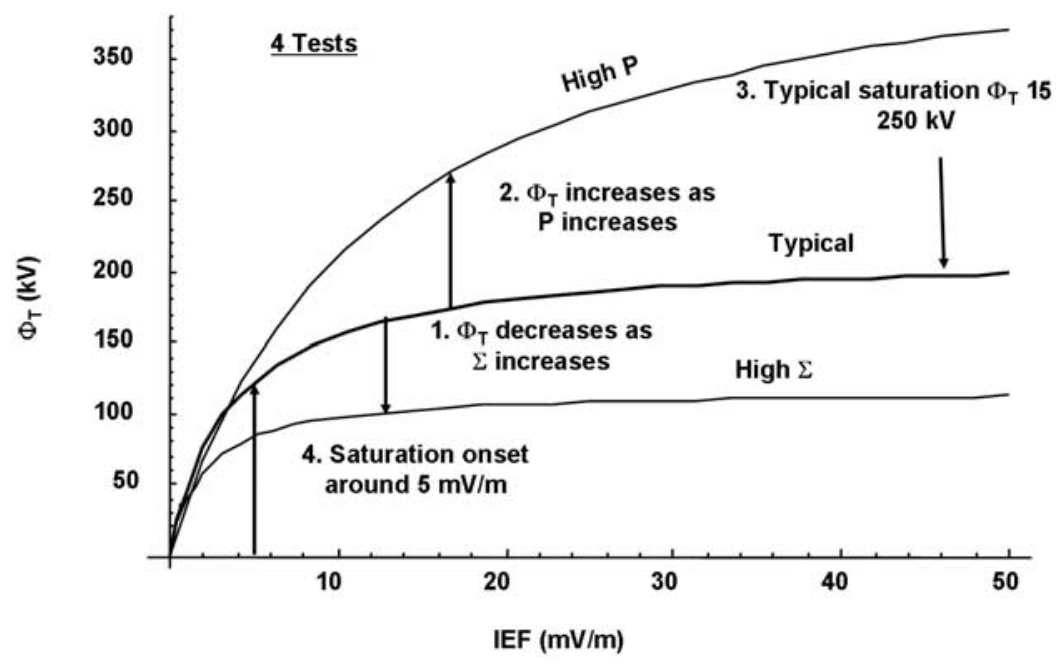

Figure 5. Dependence as predicted by the Hill model of the transpolar potential on the interplanetary electric field for a southward pointing interplanetary magnetic field. The three curves illustrate the variation in this dependence that occurs on increasing solar wind ram pressure and ionospheric conductance (modified from Siscoe et al. [2002a]).

and

$$
\Phi_{\mathrm{S}}(\mathrm{kV})=5760 \mathrm{P}(\mathrm{nPa})^{1 / 3} /(\xi \Sigma(\mathrm{S}))
$$

Equation (2) is derived from a basic reconnection model, which takes account of the rate of reconnection, the length of the reconnection line, the stagnation pressure, and dipole scaling. In each case standard representations for the quantities are used. The only empirically determined factor used is the ratio of the magnetosheath magnetic field at the stagnation point to the IMF [Crooker et al., 1982]. Equation (2) applies to the case in which the IMF points straight south. The original expression includes a standard factor to generalize it to arbitrary orientation of the IMF.

[23] Equation (3) is derived from a representation of the region 1 current system as two circles forming an upright figure eight in the dawn-dusk meridian plane and connecting the magnetopause to the Earth [Siscoe et al., 2002a, 2002b]. The standard shape of the magnetosphere is used to relate the radius of the circles to the distance to the magnetopause, and dipole scaling is used for ram pressure variation. The strength of the current in the system is set by requiring that relative to the dipole field at the stagnation point, the strength of the field it generates be the unbiased fraction $1 / 2$ discussed previously. The value of the geometrical factor $\xi$ that enters Ohm's law as $\mathrm{I}_{1}=\xi \Sigma \Phi_{\mathrm{T}}$ is obtained from MHD simulation. (It is typically between 3 and 4.) Though this is a crude model, it incorporates the scaling properties of the essential components.

[24] Combining equations (1), (2), and (3) gives the transpolar potential as a function of ram pressure, IEF, and ionospheric conductance that the Hill model as parameterized by equations (2) and (3) predicts.

$$
\begin{aligned}
\Phi_{\mathrm{T}}(\mathrm{kV})= & 57.6 \mathrm{P}(\mathrm{nPa})^{1 / 3} \mathrm{E}(\mathrm{mV} / \mathrm{m}) /\left(\mathrm{P}(\mathrm{nPa})^{1 / 2}\right. \\
& +0.01 \xi \Sigma(\mathrm{S}) \mathrm{E}(\mathrm{mV} / \mathrm{m})) .
\end{aligned}
$$

Figure 5 gives plots of $\Phi_{\mathrm{T}}$ as a function of IEF evaluated from equation (4) for both typical and high values of $\mathrm{P}$ and $\Sigma$.

[25] Figure 5 mentions four testable predictions that this formulation of the Hill mechanism makes: (1) the value of the saturation potential decreases inversely with ionospheric conductance; (2) it increases as the $1 / 3$ power of the ram pressure; (3) it typically lies between 150 and $250 \mathrm{kV}$; (4) the transition to saturation $\left(\Phi_{\mathrm{L}}=\Phi_{\mathrm{S}}\right)$ typically occurs when the driving IEF reaches about $5 \mathrm{mV} / \mathrm{m}$. To apply these tests, the following section brings together published observations and MHD simulations.

\section{Tests of Predictions Common to the Four Mechanisms}

[26] To start the tests based on predictions from equation (4) and illustrated in Figure 5, Figure 6 shows data from two storms for which the transpolar potential has been determined [Russell et al., 2000; Hairston et al., 2003]. It shows that in the saturation domain equation (4) predicts values for the transpolar potential that are closer to observations than values based on extrapolating the linear part of the curve (or any data-based linear prediction formula) into the saturation domain. Moreover, the data for the 1998 storm show that the transition to saturation occurs around the predicted value of $\sim 5 \mathrm{mV} / \mathrm{m}$. Very similar findings resulted also from statistical studies of radar data [Shepherd et al., 2002, 2003] and polar cap index data [Tsutomu, 2002].

[27] The predicted fractional power dependence on ram pressure of the saturated transpolar potential is supported indirectly by evidence that other things being about equal, the rate of energy injected into the ring current increases when the ram pressure is greater [Wang et al., 2003]. This behavior would follow from Figure 1, since if the ram pressure happens to be unusually high, the transpolar potential can saturate at a higher value and the storm can be stronger. Of course, this interpretation is not unique. It might instead be that increased ram pressure means greater 

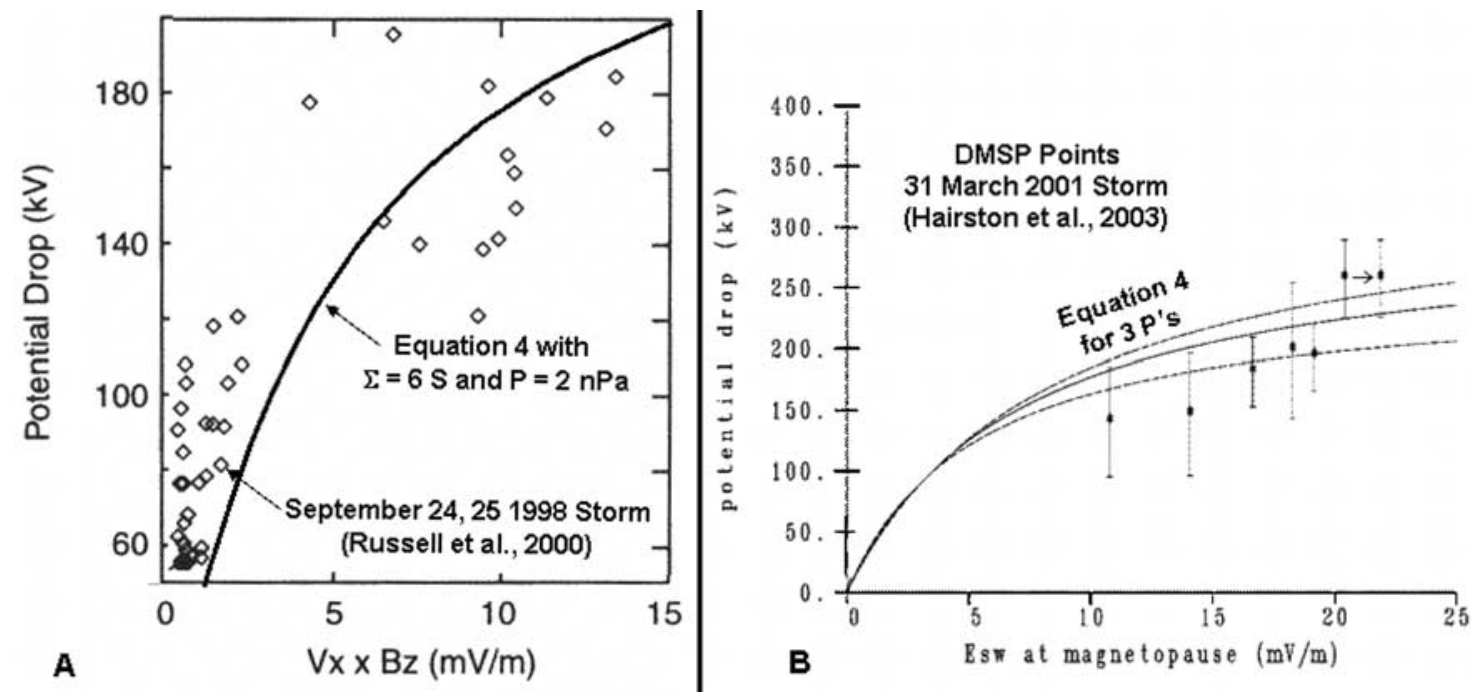

Figure 6. Two plots comparing transpolar potentials that reach saturation with values calculated from equation (4).

solar wind density and so more ions available to make the ring current [Kozyra et al., 1998]. The point here is merely that the ram pressure dependence is consistent with the Hill model as well.

[28] Direct evidence in support of the predicted dependence on ionospheric conductance and ram pressure comes from case studies by Ober et al. [2003] in which values of the transpolar potentials obtained from the DMSP polarorbiting satellite were plotted against the solar wind electric field. The data were grouped into bins of high and low ram pressure for similar ionospheric conductance and into bins of high and low ionospheric conductance for similar ram pressure. Ionospheric conductance was inferred from solar $\mathrm{UV}$ as parameterized by $\mathrm{F} 10.7$ solar radio intensity [Robinson and Vondrak, 1984]. Since the orbit of the DMSP satellite rarely crossed the polar cap precisely through the peak potential difference and so most often measured less than the full potential difference, the data yielded a scatter- plot, which, to apply as a test, should have the predicted potential as an upper bound.

[29] Figure 7 shows scatterplots of transpolar potential as a function of IEF for two situations in which the average ionospheric conductances differed by a factor of 1.8 (proportional to the square root of the F10.7 intensity [Robinson and Vondrak, 1984]) while solar wind ram pressures were similar. The curves in the figure are plots of equation (4) but modified to take into account a $30 \mathrm{kV}$ background potential that exists at zero IEF, which the formulation given by equation (4) ignores. One sees that the curves form reasonable upper bounds on the data points in both the high and low conductance cases. The upper bound character of the curve is made more apparent for the low-conductance data in the right panel by showing also the high-conductance curve (dashed line) for comparison.

[30] Figure 8 shows scatterplots of transpolar potential as a function of IEF for two situations in which the average solar

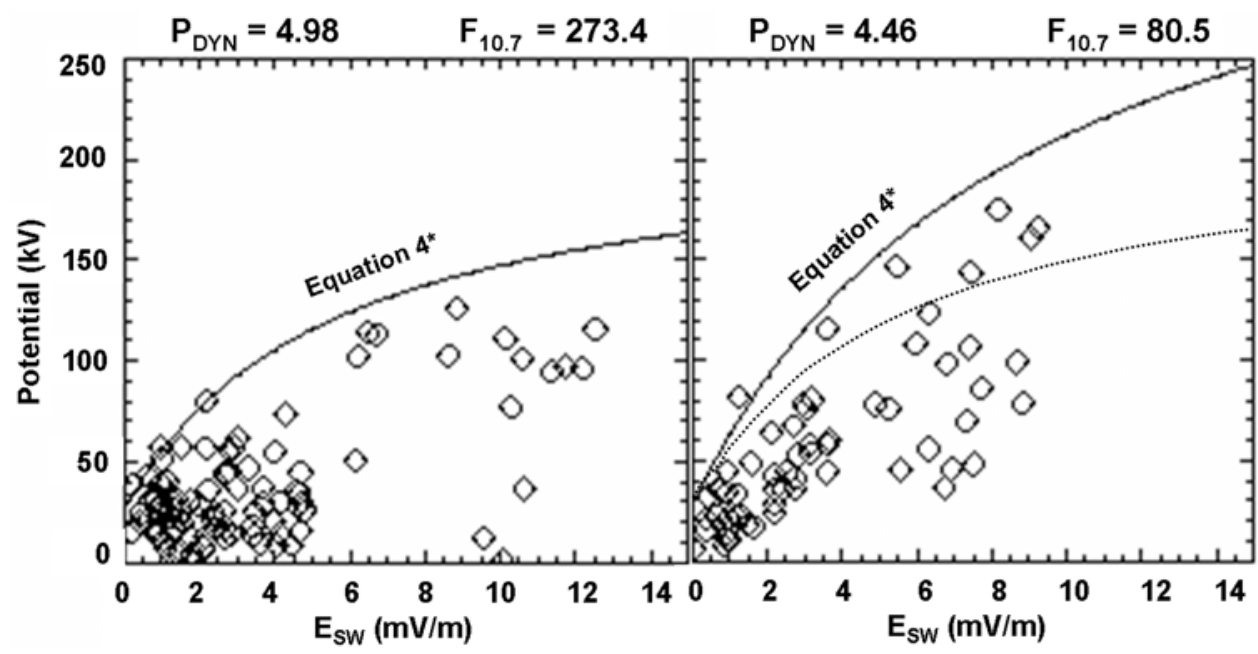

Figure 7. Scatterplots of transpolar potential measure by the DMSP satellite as a function of the interplanetary electric field (IEF) under conditions of high ionospheric conductance (left) and low ionospheric conductance (right) but similar solar wind ram pressure [Ober et al., 2003]. 


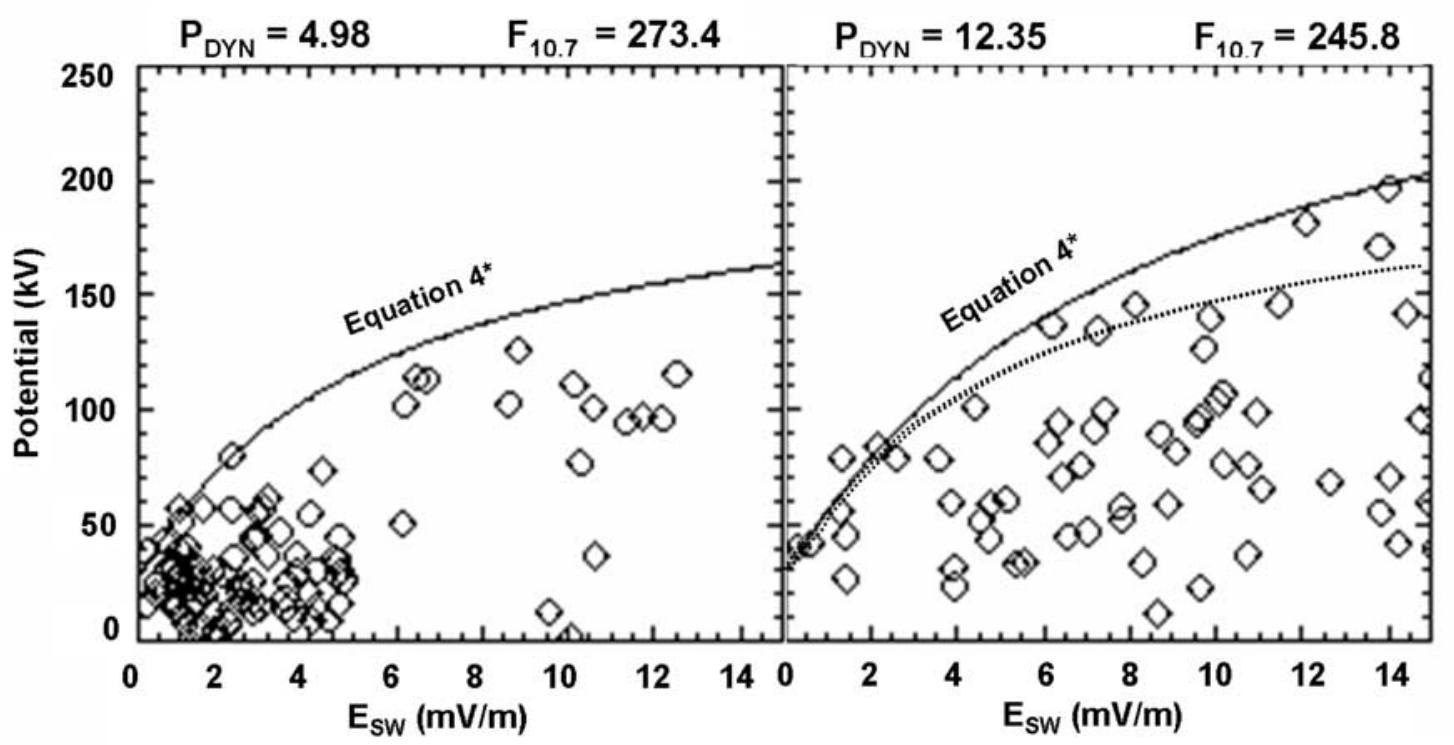

Figure 8. Scatterplots of transpolar potential measure by the DMSP satellite as a function of the IEF under conditions of low ram pressure (left) and high ram pressure (right) but similar ionospheric conductance [Ober et al., 2003].

wind ram pressures differed by a factor of 2.5 while ionospheric conductances were similar. Again the curves in the figure are plots of equation (4) modified as already noted. Here too the curves form reasonable upper bounds on the data points in both the high and low ram pressure cases. The high ram pressure case (right) has more data points at high IEF, as expected, since high IEF correlates with high solar wind speed and hence also with high ram pressure. The upper bound character of the curve is therefore more convincing in the right panel, especially by comparison with the low ram pressure curve shown in the same panel (dashed line).

[31] In contrast to the Ober et al. result shown in Figure 8, Shepherd et al. [2003], in a statistical study that used synoptic radar observations to determine the transpolar potential, reports no statistically significant variation in the of average transpolar potential over a factor of 10 variation in ram pressure. The Shepherd et al. study had, however, a maximum IEF bin range of $4 \mathrm{mV} / \mathrm{m}$ to $5 \mathrm{mV} / \mathrm{m}$. If one terminates the Ober et al. plots at $5 \mathrm{mV} / \mathrm{m}$, they too would show no significant dependence on ram pressure. So it is perhaps too early to worry that the observations disagree on this point.

[32] MHD simulations can also be used to carry out the four tests specified in Figure 5. Test 1 asks whether the saturated transpolar potential decreases with increasing ionospheric conductance. Figure 9 compares four MHD simulations against predicted dependence on conductivity as parameterized by equation (4). In the simulation the driving IEF is $1.75 \mathrm{mV} / \mathrm{m}$, the ram pressure is $1 \mathrm{nPa}$, and the ionospheric conductance is uniform. The test spans from the linear to the saturated domain as the conductance increases from 2 to 44 Siemens $\left(\Phi_{\mathrm{L}} / \Phi_{\mathrm{S}}\right.$ varies from 0.12 to 2.6). Agreement between the prediction and the simulations is quite good. Results shown in Figure 9 were obtained with the ISM MHD code, and similar dependence of the saturated transpolar potential has been reported with the University of Michigan code [Ridley, 2002].
[33] To address test 2 (the increase of saturation potential with increasing ram pressure), two simulations in the saturation domain were performed with southward IMF and IEF $=10 \mathrm{mV} / \mathrm{m}$ in each case but differing by a factor of two in ram pressure $(2 \mathrm{nPa}$ and $4 \mathrm{nPa})$. Between the lower and higher ram pressure cases, the transpolar potential increases from $144.4 \mathrm{kV}$ to $168.2 \mathrm{kV}$. The ratio is 1.16 . For the same parameters used by the code, equation (4) predicts a ratio of 1.14 . This test therefore corroborates the ram pressure prediction of the Hill model.

[34] To perform tests 3 and 4 in Figure 1 (shape of the curve and actual values of the predicted transpolar potential), Figure 10 compares predictions of equation (4) against the transpolar potential computed and by four MHD simulation runs and against observations taken during a magnetic storm. The four MHD runs span from the linear domain into the saturation domain. The lower panel in the figure shows transpolar potentials measured during the magnetic storm 2 October 2001 (plus signs) and corresponding values

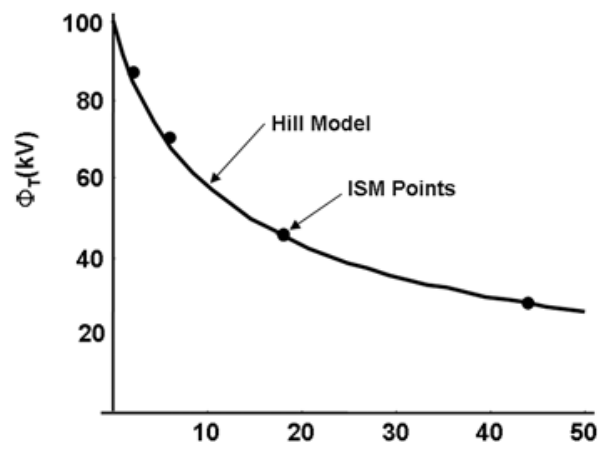

Figure 9. Showing the dependence of transpolar potential on changes in ionospheric conductance as predicted by the Hill model (as equation (4)) and computed by MHD simulation (from Siscoe et al. [2002a]). 


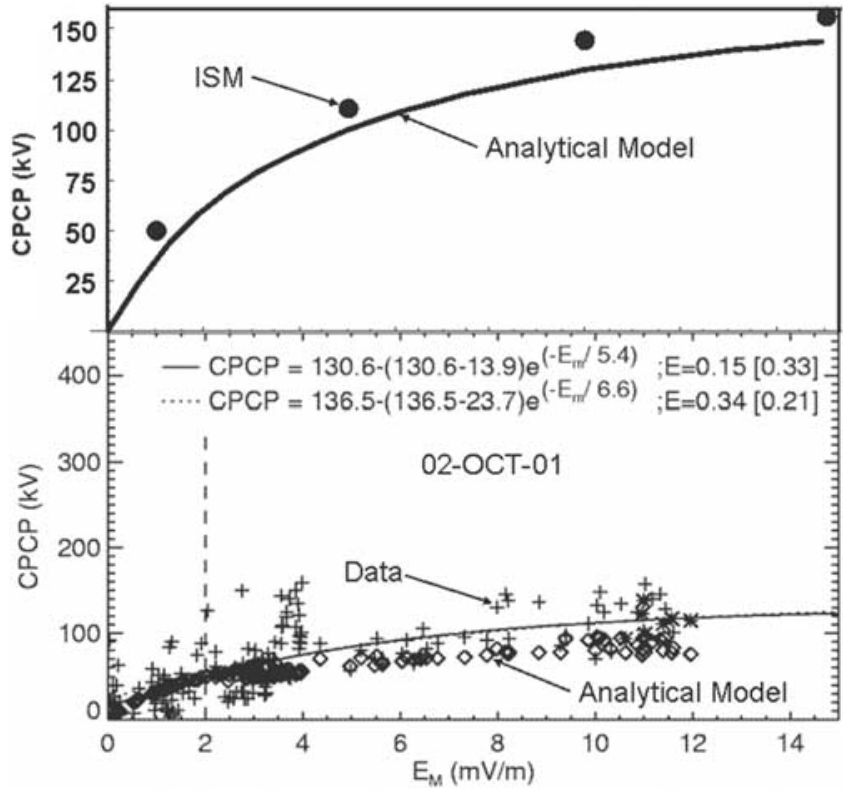

Figure 10. (top) Dependence of transpolar potential (labeled CPCP in this figure) on solar wind electric field (southward IMF) as predicted by the analytical parameterization of equation (4) and as computed by MHD simulation (the ISM code) (from Siscoe et al. [2002a]). (bottom) For the storm of 2 October 2001, transpolar potential inferred directly from measurements (plus signs) and as calculated from the analytic formation using corresponding measurements (diamonds). The curves show analytical fits to the data points as specified at the top of the plot (from Ridley [2002]).

calculated from the analytical representation of the Hill model under test [Ridley, 2002]. In both panels, values calculated with the Hill model are slightly less than the comparison values. The agreement is nonetheless reasonably good in terms of both shape and magnitude. For instance, in both cases the transition to the saturation domain occurs over a stretch of IEFs that centers on approximately $5 \mathrm{mV} / \mathrm{m}$.

[35] More needs to be said about the bottom panel. It shows one example from a set of similarly prepared plots for several different storms [Ridley, 2002] in each of which, as here, there is reasonable agreement between the calculated and measured potentials. In addition, for some storms in the set the potential versus IEF curve exhibits no marked tendency toward saturation despite the IEF reaching levels between 15 and $20 \mathrm{mV} / \mathrm{m}$, which, one would think, should be in the saturation domain. This could be a manifestation of the effect of ram pressure on saturation. Other things being equal, the value of the IEF needed to enter the saturation domain (as measured by the value at which the potential reaches $1 / 2$ of the asymptotic saturation value) increases as $\mathrm{p}^{1 / 2}$. So, for example, a factor of 10 increase in ram pressure would move the threshold for saturation from around $5 \mathrm{mV} / \mathrm{m}$ to around $16 \mathrm{mV} / \mathrm{m}$, as can be seen in Figure 1, which might give the impression of little or no saturation.

[36] To sum up this section, the four mechanisms seem to have passed their common set of tests reasonably well. Recall that this merely means that the tests confirm a central role for the region 1 current system for each mechanism and that this role becomes apparent in each of them when the region 1 current system generates a magnetic field that is comparable in strength to the dipole field at the dayside magnetopause. The tests apply indirectly to the massloading mechanism of Winglee et al. [2002], in that the criterion on the strength of the region 1 field that has just been tested does not obviously apply to the mass-loading mechanism. Before the tests can be applied to the massloading mechanism, its altogether different relation to the region 1 current system must be made explicit so that predictions can be made. It seems unlikely that the predictions would be the same as given by equation (4), which have been tested here.

\section{Summary}

[37] Transpolar potential saturation appears to be a wellobserved fact. This paper has compared four mechanisms put forward to explain it: (1) significant weakening of the magnetic field at the stagnation point, which limits the rate of magnetic reconnection; (2) formation of a dimple, which chokes the flow into the reconnection line; (3) widening of the magnetosheath, which lets the solar wind flow around the magnetosphere with less aid from magnetic reconnection; and (4) usurpation of the Chapman-Ferraro current by the region 1 current system, which limits the total region 1 current to the value which stops the solar wind and deflects it around the magnetosphere. The prerequisite assumptions behind these mechanisms (weakened field, dimple formation, widened magnetosheath, and usurped current) are supported by MHD simulations. The models have in common a significant role for the region 1 current system. An attempt to use the region 1 current system to devise a test to determine which mechanism is really operative fails because the role that the region 1 current system plays turns out to be virtually indistinguishable between them, at least at the present level of the mechanisms' formulation. This being so, one can take advantage of a parameterization that has been worked out for mechanism 1 to test the four mechanisms as a group. The parameterization makes four testable predictions: (1) the value of the interplanetary electric field at which saturation should become evident, (2) the value of the transpolar potential at saturation, (3) the dependence of the transpolar potential on solar wind ram pressure, and (4) the dependence of the transpolar potential on ionospheric conductance. Applying these four tests by making comparison with available observations and MHD simulations shows that the mechanisms as a group seem to pass reasonably well. However, the tests cannot be said to be definitive. More observations and simulations should be made and brought to bear on the issue.

[38] Acknowledgments. This work was supported in part by the National Science Foundation under grant ATM-0220396. Work at UCLA and UNH was supported in part by the National Science Foundation under grant ATM-0097143. Work at the University of Michigan was supported by the DoD MURI program (grant F4960-01-1-0359).

[39] Arthur Richmond thanks K. Papadopoulos and another reviewer for their assistance in evaluating this paper.

\section{References}

Cahill, L. J., Jr., and J. R. Winckler (1999), Magnetopause crossings observed at 6.6 RE, J. Geophys. Res., 104, 12,229-12,237. 
Crooker, N. U., G. L. Siscoe, P. R. Mullen, C. T. Russell, and E. J. Smith (1982), Magnetic field compression at the dayside magnetopause, J. Geophys. Res., 87, 10,407-10,412.

Hairston, M. R., T. W. Hill, and R. A. Heelis (2003), Observed saturation of the ionospheric polar cap potential during the 31 March 2001 storm, Geophys. Res. Lett., 30(6), 1325, doi:10.1029/2002GL015894.

Hill, T. W., A. J. Dessler, and R. A. Wolf (1976), Mercury and Mars: The role of ionospheric conductivity in the acceleration of magnetospheric particles, Geophys. Res. Lett., 3, 429-432.

Janhunen, P., H. E. J. Koskinen, and T. I. Pulkkinen (1996), A new global ionosphere-magnetosphere coupling simulation utilizing locally varying time step, in Proceedings of the Third International Conference on Substorms (ICS-3), EAS SP-389, pp. 205-210, Eur. Space Agency, Paris.

Kozyra, J. U., V. K. Jordanova, J. E. Borovsky, M. F. Thomsen, D. J. Knipp, D. S. Evans, D. J. McComas, and T. E. Cayton (1998), Effects of a high-density plasma sheet on ring current development during the November 2-6, 1993, magnetic storm, J. Geophys. Res., 103, 26,28526,305 .

Liemohn, M. W., J. U. Kozyra, M. R. Hairston, D. R. Weimer, G. Lu, A. J. Ridley, T. H. Zerbuchen, and R. M. Skoug (2002), Consequences of a saturated convection electric field on the ring current, Geophys. Res Lett., 29(9), 1348, doi:10.1029/2001GL014270.

Merkine, V. G., K. Papadopoulos, G. Milikh, A. S. Sharma, X. Shao, J. Lyon, and C. Goodrich (2003), Effects of the solar wind electric field and ionospheric conductance on the cross polar cap potential: Results of global MHD modeling, Geophys. Res. Lett., 30(23), 2180, doi:10.1029/ 2003GL017903.

Ober, D. M., N. C. Maynard, and W. J. Burke (2003), Testing the Hill model of transpolar potential saturation with observations, J. Geophys. Res., 108(12), 1467, doi:10.1029/2003JA010154.

Raeder, J., and G. Lu (2004), Polar cap potential saturation during large geomagnetic storms, Adv. Space Res., in press.

Raeder, J., Y. L. Wang, T. J. Fuller-Rowell, and H. J. Singer (2001), Global simulation of space weather effects of the Bastille Day storm, Sol. Phys., 204, 325.

Ridley, A. (2001), BATS-R-US simulation of CPVP, paper presented at GEM Mini-workshop, Natl. Sci. Found., San Francisco, Calif

Ridley, A. (2002), A possible cause for the saturation of the CPCP, paper presented at GEM Summer Workshop, Natl. Sci. Found., Telluride, Colo.

Ridley, A. (2003), The saturation of the ionospheric cross polar cap potential and the magnetospheric configuration during extreme solar wind and IMF conditions, paper presented at Yosemite Conference-Workshop: The Dayside Magnetosphere and Cusp, NASA, Yosemite Natl. Park, Calif.

Robinson, R. M., and R. R. Vondrak (1984), Measurements of E region ionization and conductivity produced by solar illumination at high latitudes, J. Geophys. Res., 89, 3951-3956.

Russell, C. T., G. Lu, and J. G. Luhmann (2000), Lessons from the ring current injection during the September 24, 25, 1998 storm, Geophys. Res. Lett., 27, 1371-1374.

Russell, C. T., J. G. Luhmann, and G. Lu (2001), Nonlinear response of the polar ionosphere to large values of the interplanetary electric field, J. Geophys. Res., 106, 18,496-18,504.
Shepherd, S. G., R. A. Greenwald, and J. M. Ruohoniemi (2002), Cross polar cap potentials measured with Super Dual Auroral Radar Network during quasi-steady solar wind and interplanetary magnetic field conditions, J. Geophys. Res., 107(A7), 1075, doi:10.1029/2001JA000109.

Shepherd, S. G., J. M. Ruohoniemi, and R. A. Greenwald (2003), Testing the Hill model of transpolar potential with Super Dual Auroral Radar Network observations, Geophys. Res. Lett., 30(1), 1002, doi:10.1029/ 2002GL015426.

Siscoe, G. L., N. U. Crooker, G. M. Erickson, B. U. Ö. Sonnerup, K. D. Siebert, D. R. Weimer, W. W. White, and N. C. Maynard (2000), Global geometry of magnetospheric currents, in Magnetospheric Current Systems, Geophys. Monogr. Ser., vol. 118, edited by S.-I. Ohtani, pp. 41-52, AGU, Washington, D. C.

Siscoe, G. L., G. M. Erickson, B. U. Ö. Sonnerup, N. C. Maynard, J. A. Schoendorf, K. D. Siebert, D. R. Weimer, W. W. White, and G. R. Wilson (2002a), Hill model of transpolar potential saturation: Comparisons with MHD simulations, J. Geophys. Res., 107(A6), 1075, doi:10.1029/ 2001JA000109.

Siscoe, G. L., N. U. Crooker, and K. D. Siebert (2002b), Transpolar potential saturation: Roles of region 1 current system and solar wind ram pressure, J. Geophys. Res., 107(A10), 1321, doi:10.1029/2001JA009176.

Spreiter, J. R., and A. L. Summers (1967), On conditions near the neutral points on the magnetosphere boundary, Planet. Space Sci., 15, 787-798.

Tanaka, T. (1995), Generation mechanisms for magnetosphere-ionosphere current systems deduced from a three-dimensional MHD simulation of the solar wind-magnetosphere-ionosphere coupling process, J. Geophys. Res., 100, 12,057-12,074.

Tsutomu, N. (2002), Saturation of polar cap potential by intense solar wind electric fields, Geophys. Res. Lett., 29(10), 1422, doi:10.1029/ 2001GL014202.

Wang, C. B., J. K. Chao, and C.-H. Lin (2003), Influence of the solar wind dynamic pressure on the decay and injection of the ring current, J. Geophys. Res., 108(A9), 1341, doi:10.1029/2003JA009851.

White, W. W., J. A. Schoendorf, K. D. Siebert, N. C. Maynard, D. R. Weimer, G. L. Wilson, B. U. Ö. Sonnerup, G. L. Siscoe, and G. M. Erickson (2001), MHD simulation of magnetospheric transport at the mesoscale, in Space Weather, Geophys. Monogr. Ser., vol. 125, edited by P. Song et al., pp. 229-240, AGU, Washington, D. C.

Winglee, R. M., D. Chua, M. Brittnacher, G. K. Parks, and G. Lu (2002) Global impact of ionospheric outflows on the dynamics of the magnetosphere and cross-polar cap potential, J. Geophys. Res., 107(A9), 1237, doi:10.1029/2001JA000214

J. Raeder, Space Science Center, University of New Hampshire, 39 College Road, Durham, NH 03824, USA. (j.raeder@unh.edu)

A. J. Ridley, Space Physics Research Laboratory, University of Michigan, 2455 Hayward Street, Ann Arbor, MI 48109-2143, USA. (ridley@umich. edu)

G. Siscoe, Center for Space Physics, Boston University, 725 Commonwealth Avenue, Boston, MA 02215, USA. (siscoe@bu.edu) 